\title{
Different Categories of Quantum Fields
}

\author{
E. Comay \\ Charactell Ltd., Tel-Aviv, Israel \\ Email: elicomay@tauex.tau.ac.il
}

How to cite this paper: Comay, E. (2019) Different Categories of Quantum Fields. Open Access Library Journal, 6: e5802. https://doi.org/10.4236/oalib.1105802

Received: September 18, 2019

Accepted: October 11, 2019

Published: October 14, 2019

Copyright $\odot 2019$ by author(s) and Open Access Library Inc.

This work is licensed under the Creative Commons Attribution International License (CC BY 4.0).

http://creativecommons.org/licenses/by/4.0/

\begin{abstract}
Properties of a quantum field that represents an elementary particle and a quantum field that mediates an interaction between particles are analyzed. This analysis relies on fundamental physical principles. The mathematical structure of these fields proves that they are completely different physical objects. A further analysis proves that a quantum field that represents an elementary massive particle and a quantum field that represents a massless particle have a completely different mathematical structure. The results are used in an examination of free spin-1/2 elementary massive particles and other free elementary particles that have an integral spin. Inherent inconsistencies are found for elementary massive particles that have an integral spin and for the Majorana neutrino. The analysis also proves that interaction mediating fields do not represent a genuine particle.
\end{abstract}

\section{Subject Areas}

Quantum Mechanics

\section{Keywords}

Quantum Field Theories, The Variational Principle, Fields of Elementary Particles

\section{Introduction}

The primary objective of a physical theory is to provide an acceptable description of experimental results that belong to its domain of validity. This criterion means that if a given theory fails to appropriately describe results of such an experiment then it should be rejected. Furthermore, physics has already acquired the status of a mature science and its theories take a mathematical structure. This issue means that mathematics provides another kind of acceptability criterion for a physical theory-it must be mathematically flawless.

Field theories play an important role in physics. The present work analyzes 
some fields that are used in quantum theories. Important information is obtained from Wigner's analysis of the irreducible representations of the Poincare group. This analysis proves that massive particles and massless particles have inherently different properties [1] [2] [3] [4]. Therefore, quantum fields of massive particles and quantum fields of massless particles are analyzed separately.

This work shows that experimental evidence and well-established theoretical principles are relevant to an examination of the validity of the mathematical structure of several field theories. For example, it is now recognized that the variational principle plays a primary role in the structure of quantum theories (see e.g. [2], chapter 7). This principle uses a Lagrangian density that depends on quantum functions of the form $\psi(x)$, where $x$ denotes a single set of four space-time coordinates $x \equiv(t, x, y, z)$. The function $\psi(x)$ describes properties of the quantum particle. For instance, the density of a Dirac particle is $\rho=\psi^{\dagger}(x) \psi(x)$. This form of $\psi(x)$ means that this function can provide information on the probability $\rho$ of finding the quantum particle at $x$, but it cannot describe its distribution around $x$, because such a distribution requires more degrees of freedom. For this reason, the present work examines elementary pointlike quantum particles. The measured upper bound of the electron's radius is about 7 orders of magnitude smaller than the proton's radius [5]. This is an amazing example of the pointlike attribute of an elementary quantum particle, and of the suitability of the above mentioned form of its Lagrangian density.

This work examines simple states of elementary particles, namely free particles, their corresponding interactions and the fields associated with these interactions. The interaction of a given free particle can be turned on continuously, for example by moving the interaction source from infinity to the particle's vicinity. The present particle classification [6] shows that particles that satisfy these conditions are the three charged leptons, $e, \mu, \tau$, their neutrinos, the corresponding antiparticles, and also the photon and the $W^{ \pm}, Z$ and the Higgs particles.

Units where $\hbar=c=1$ are used. Therefore, just one dimension is required and the dimension of length $[L]$ is used. The Minkowski metric $g_{\mu \nu}$ is diagonal and its entries are $(1,-1,-1,-1)$. Relativistic expressions are written in the standard notation. Section 2 describes several constraints that apply to quantum theories. Section 3 describes the relevance of these constraints to the consistency of quantum fields of the above mentioned particles. Some features of the results are discussed in the fourth section. The last section summarizes the main points of this paper.

\section{Constraints on Field Theories}

The following items explain how well-established physical principles yield acceptability criteria that apply to every quantum theory.

C.1) Special relativity is a valid theory. The operation of modern accelerators and the tremendous amount of their data provide a solid experimental support 
for special relativity. Hence, expressions should take a relativistic covariant form.

C.2) As stated above, the variational principle is regarded as a solid basis for a quantum field theory of an elementary particle (see e.g. [2], chapter 7). In particular, an adequate Lagrangian density satisfies many conservation laws. This issue is described in the following textbook which states that this principle provides "the foundation on which virtually all modern theories are predicated" (see [7], p. 353). Therefore, a consistent Lagrangian density that yields the theory's equations of motion is required for every specific quantum theory. It turns out that "all field theories used in current theories of elementary particles have Lagrangians of this form" (see [2], p. 300):

$$
\mathcal{L}_{P}=\mathcal{L}\left[\psi(x), \partial \psi(x) / \partial x^{\mu}\right],
$$

where the subscript ${ }_{P}$ denotes that $\mathcal{L}$ describes a specific particle, and $x$ denotes the four space-time coordinates. The action

$$
S=\int \mathcal{L} \mathrm{d}^{4} x
$$

has the dimension of $\hbar$. It follows that in the units used herein, the dimension of a Lagrangian density is $\left[L^{-4}\right]$.

C.3) The de Broglie formula for the wave length of a free massive particle is related to its momentum (see [8], p. 52 or [9], p. 3)

$$
\lambda=2 \pi \hbar / p \text {. }
$$

This relation is assumed to be correct.

C.4) Wigner has analyzed the irreducible representations of the inhomogeneous Lorentz group [1] [2] [3] [4]. Important results of his work show that a massive quantum particle has a well-defined mass and spin. A massless particle belongs to a different class. By definition, it has a zero mass and it moves at the speed of light in every inertial frame. Instead of spin, it has two components of helicity.

C.5) A field theory, like any other physical theory, must yield quantities that are related to results of a measurement. Established laws of physics require "connections between the results of observations, on one hand, and the equations of the mathematical formalism on the other" (see [10], p. 34). In a theory of a particle that is derived from the variational principle, this goal is obtained from two kinds of terms: terms that determine the time evolution of a free particle and interaction terms that induce changes in the particle's time evolution that result from the presence of external fields. Therefore, a consistent interaction term is an indispensable element of a field theory. The interaction involves two kinds of fields: a field that represents the quantum particle (called $Q_{1}$ ) and a field that mediates a specific interaction which affects the state of $Q_{1}$. Evidently, the interaction can be turned on continuously, for example by moving the interaction source from infinity to the particle's vicinity. An interaction that is expressed by a specific term of the Lagrangian density satisfies this requirement. Thus, the general form of the Lagrangian density of an interacting particle is an extension of its free Lagrangian density (1) 


$$
\mathcal{L}_{\text {full }}=\mathcal{L}_{\text {free }}\left[\psi(x), \partial \psi(x) / \partial x^{\mu}\right]+\mathcal{L}_{\text {int }}\left[\psi(x), \partial \psi(x) / \partial x^{\mu}, \chi(x), \partial \chi(x) / \partial x^{\mu}\right],
$$

where $\chi$ denotes the interaction carrying field.

C.6) Correspondence relationships exist between the following four theories:

$$
\mathrm{QFT} \leftrightarrow \mathrm{RQM} \leftrightarrow \mathrm{QM} \leftrightarrow \mathrm{NCM} .
$$

(QFT denotes Quantum Field Theory; RQM denotes Relativistic Quantum Mechanics; QM denotes Quantum Mechanics; NCM denotes Nonrelativistic Classical Mechanics.) The important relationships between the first three theories are stated in a well-known textbook: "First, some good news: quantum field theory is based on the same quantum mechanics that was invented by Schroedinger, Heisenberg, Pauli, Born, and others in 1925-26, and has been used ever since in atomic, molecular, nuclear and condensed matter physics" (see [2], p. 49). Below, these relationships are called the Weinberg correspondence principle. The last relationship is known as the classical limit (see e.g. [8], chapter VI; [9], pp. 25-27, 137, 138).

The Hilbert space is an important element of QM (see [2], pp. 49, 50). A useful basis of a Hilbert space comprises orthonormal functions that satisfy

$$
\int \psi_{i}^{\dagger} \psi_{j} \mathrm{~d} \tau=\delta_{i j},
$$

where $\mathrm{d} \tau$ denotes a volume element and $\delta_{i j}$ denotes the Kronecker delta (see [8], pp. 164, 165). Operators of quantum mechanics cast an element of the Hilbert space to one or more elements of this space.

C.7) Evidently, the intensity of an interaction mediating field is not uniform throughout the entire universe. Hence, the strength of an interaction depends on specific values of this field at the particle's location. The probability of finding a quantum particle at a given spatial volume is proportional to its density.

The Noether theorem yields a consistent expression for the density of a quantum particle. Consider a Lagrangian density that is invariant under a variation of a global phase factor $\exp (i \alpha)$. Applying a small variation $\alpha \neq 0$ to the Lagrangian density, one obtains the following quantities which are proportional to $\alpha \quad$ (see [11], p. 314)

$$
0=i \alpha\left[\frac{\partial \mathcal{L}}{\partial \psi}-\partial_{\mu}\left(\frac{\partial \mathcal{L}}{\partial\left(\partial_{\mu} \psi\right)}\right)\right] \psi+i \alpha \partial_{\mu}\left(\frac{\partial \mathcal{L}}{\partial\left(\partial_{\mu} \psi\right)} \psi\right)
$$

The expression inside the square brackets of (7) vanishes due to the Euler-Lagrange equation of the system. This is an example that shows the crucial role of the system's equation of motion. The second term yields the Noether expression for a conserved 4-current

$$
j^{\mu}=\frac{\partial \mathcal{L}}{\partial\left(\partial_{\mu} \psi\right)} \psi,
$$

and of its continuity equation

$$
0=j_{, \mu}^{\mu}=\partial_{\mu}\left(\frac{\partial \mathcal{L}}{\partial\left(\partial_{\mu} \psi\right)} \psi\right) .
$$


This expression shows that the conserved 4-current and its density component $j^{0}$ are derived from terms of the Lagrangian density that contains derivatives of the quantum function $\partial \psi(x) / \partial x^{\mu}$.

An examination of the full Lagrangian density of a quantum particle (4) shows that if the interaction term $\mathcal{L}_{\text {int }}$ depends on derivatives $\partial \psi(x) / \partial x^{\mu}$ then the original density changes, and the new density also depends on the external field $\chi(x)$ and/or its derivatives $\partial \chi(x) / \partial x^{\mu}$. It means that density cannot be consistently constructed in this case. Negative aspects of this kind of derivatives are already recognized in the literature, where it is stated that problems "appear with a vengeance" (see [12], p. 87).

Conclusion: An interaction term that depends on derivatives of the quantum function is unacceptable because it destroys the original expression for density as well as the inner product of the Hilbert space. This conclusion also holds for the Fock space, because this space is an extension of the Hilbert space to the case of more than one identical particle (see [13], pp. 40-41).

C.8) This item emphasizes the crucial role of differential equations in the structure of quantum theories. Evidently, the measurability requirement 5 depends on a time-dependent process which shows a change of the measuring device from an initial state at an initial time to a final state at a later time. Therefore, a satisfaction of this requirement depends on a consistent time-dependent expression that describes the system's evolution. Solutions of differential equation are used for this purpose.

Special relativity relates a space-time interval in one frame to a different space-time interval in another frame. It follows that the required differential equation is a partial differential equation with respect to the four space-time coordinates.

Furthermore, requirement 2 points out the significance of the variational principle as a basis for a quantum field theory. The Noether theorem provides a strong support for this issue because it connects symmetries with conservation laws. In this way one can be sure that an appropriate symmetry of a given theory means that this theory abides by the required conservation law. Requirement 7 shows how the Noether theorem provides an expression for a conserved 4-current. Similarly, it can be proved that conservation of energy-momentum and of angular momentum are derived from a Lagrangian density that does not depend explicitly on space-time coordinates (see [12], pp. 17-19). It turns out that the Euler-Lagrange equations of motion of the system are a crucial element of the proof of the above mentioned applications of the Noether theorem.

The following examples illustrate this issue. Maxwell equations of electromagnetic fields and Dirac equation of massive spin-1/2 particles take the form of partial differential equations with respect to the four space-time coordinates. These equations are the Euler-Lagrange equations that are derived from an appropriate Lagrangian density (see [12], p. 54; [14], pp. 78, 79). These equations successfully explain the time-evolution of these fields, and provide a solid theoretical basis for modern technology. 
Conclusion: the differential equations of the system are an indispensable element of a quantum theory.

\section{Theoretical Properties of Quantum Fields}

The following discussion uses the above mentioned constraints in an examination of the compatibility of quantum theories. These theories are grouped in categories according to properties of the particles that are described by the quantum field.

\subsection{Fields of Massive Spin-1/2 Particles}

The Lagrangian density of an electrically charged spin-1/2 particle is (see [12], p. 84 ; [15], p. 78)

$$
\mathcal{L}=\bar{\psi}\left[\gamma^{\mu} i \partial_{\mu}-m\right] \psi-e \bar{\psi} \gamma^{\mu} \psi A_{\mu} .
$$

Here $\bar{\psi}=\psi^{\dagger} \gamma^{0}$ (see [16], p. 24). This is the Dirac Lagrangian density, and it is linear in the mass $m$. Hence, the $\left[L^{-4}\right]$ dimension of a Lagrangian density entails that the dimension of the product $\bar{\psi} \psi$ is $\left[L^{-3}\right]$. The Noether expression for the 4-current (8) yields the following 4-current of a Dirac particle (see [12], p. 97; [15], pp. 50, 51)

$$
j^{\mu}=\bar{\psi} \gamma^{\mu} \psi .
$$

Relation (11) is consistent with requirement 7, because it does not depend on derivatives of $\psi$. It means that the introduction of the well-known substitution of the electromagnetic interaction (see [12], p. 84; [14], pp. 47-49; [17])

$$
p_{\mu} \rightarrow p_{\mu}-e A_{\mu}
$$

yields an interaction term that is proportional to the external 4-potential $A_{\mu}$. The form of the last term of (10) demonstrates this attribute. These properties of a Dirac field are consistent with the constraints of Section 2.

Remark: The mathematically real form of the Dirac-like Majorana equation is discusses later in this section.

\subsection{Electromagnetic Fields}

Maxwellian electrodynamics is regarded as a well-established theory that is used as a reliable element of the present technology. The following discussion analyzes simple cases of electromagnetic interactions: an interference of radiation fields, and interactions of the hydrogen atom whose properties are adequately described by the Schroedinger and the Dirac equations. The Weinberg correspondence principle indicates that the following arguments also apply to QFT. The analysis examines separately radiation fields and bound fields. The validity of this distinction is derived from the linearity of Maxwell equations.

Electromagnetic radiation fields satisfy the homogeneous Maxwell equations throughout the entire space-time (see [14], pp. 116, 117)

$$
F_{, v}^{\mu v}=0, \quad F_{, v}^{* \mu v}=0,
$$


where $F^{* \mu v}=\epsilon^{\mu v \alpha \beta} F_{\alpha \beta}$, and $\epsilon^{\mu v \alpha \beta}$ is the completely antisymmetric unit tensor of the fourth rank. The null value on the right-hand side of (13) demonstrates the following self-evident argument: electromagnetic radiation (a real photon) cannot depend on a single charge simply because its two Equations (13) are charge-independent.

Figure 1 depicts two radiating systems which illustrate this conclusion. The charge $Q$ of Figure 1(A) moves uniformly along a circle embedded in the $(x, y)$ plane. This charge emits radiation due to its acceleration towards the circle's center. The point $P$ lies on the $z$-axis at the radiation zone. Let us examine a small circle around $P$ which is parallel to the $(x, y)$ plane. A nonvanishing amount of radiation (photons) flows through this circle. Figure $1(B)$ depicts two moving charges $\pm Q$. These charges are at two antipodal points, and they move like the charge of Figure 1(A). Well-known formulas of radiation fields (see [14], pp. 184-187; [18], pp. 654-658) prove that at point $P$, the fields of the charges $\pm Q$ are the same. Therefore, at point $P$ the radiation fields of Figure 1(B) are twice as strong as the radiation fields of Figure 1(A).

The Poynting vector

$$
\boldsymbol{S}=\boldsymbol{E} \times \boldsymbol{B} / 4 \pi
$$

represents energy flux (see [14], p. 81; [18], p. 237). Therefore, the energy flux of Figure 1(B) is four times greater than that of Figure 1(A). Since the charge velocity of the two systems is the same, one finds that the radiation's frequency of the two systems is the same. Hence, the number of photons of Figure 1(B) is four times greater than that of Figure 1(A). This example proves that a photon cannot be assigned to a single charge of its radiating system. This conclusion confirms the charge-independence of the homogeneous Equation (13) of electromagnetic radiation.

The mathematical structure of bound fields is not the same as that of radiation fields. Indeed, bound fields satisfy the inhomogeneous Maxwell equation (see [14], p. 79)

$$
F_{, v}^{\mu v}=-4 \pi j^{\mu} .
$$

The following discussion shows that radiation fields and bound fields are not identical physical objects. Well-known properties of two electromagnetic systems yield two independent proofs of this claim.

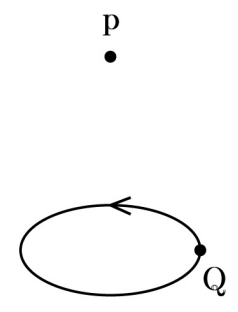

(A)

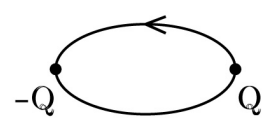

(B)

Figure 1. (A) One radiating charge. (B) Two radiating charges (See text). 
First, consider the hydrogen atom. Closed physical systems conserve angular momentum. Moreover, electrodynamic processes conserve parity. Therefore, each atomic state has a well-defined spin and parity (see e.g. [8], p. 418; [16], p.57). Consider the ground state of the hydrogen atom and an incoming photon whose energy equals the difference between the states $2 p$ and $1 s$. This is an allowed transition (see [9], p. 264) and the hydrogen atom is excited to the $2 p$ energy level. This process proves that the photon's angular momentum and parity are $1^{-}$. (The photon is massless and its angular momentum takes only the two components of helicity.) These values of the angular momentum and the parity of the photon are documented in the annual report of the Particle Data Group [6].

Let us examine bound fields of states of the hydrogen atom. Here atomic spin and parity are determined by the electronic state and the electromagnetic bound fields are completely ignored (see e.g. [16], p. 57). It means that if bound fields represent a particle then the spin-parity of such a particle is $0^{+}$. By contrast, it is shown above that the photon's spin-parity is $1^{-}$. The different spin of radiation fields and of bound fields, and the Wigner constraint 4 prove the claim that bound fields and radiation fields are different physical objects.

Let us take another kind of electromagnetic interaction-a scattering process where the mediating fields are bound fields of two colliding particles (see Figure 2). An electron $e$ moves from left to right and is scattered by an electrically charged particle Q. e'denotes the 4-momentum of the outgoing electron and $x$ denotes the sum of the 4-momentum of all other outgoing particles.

The 4-momentum transfer $q^{\mu}$ is the difference between the 4-momentum of the incoming electron and that of the outgoing electron. $q^{\mu}$ is a spacelike 4 -vector (see [19], p. 190). On the other hand constraint 4 means that all physical particles are either massive particles whose 4-momentum is timelike or massless particles that have a null 4-momentum. It follows that in a scattering process, no genuine physical particle is exchanged between the colliding particle. This is another example showing the inherent difference between a real photon and bound fields. Furthermore, this inherent difference between interaction mediating fields and a genuine particle apply to all cases that are described by the scattering experiment of Figure 2. In the case of electromagnetic fields, this difference is (at least implicitly) recognized by the general community, and physical photons are called real photons whereas energy and momentum associated with an interaction of bound electromagnetic fields are called virtual photons (see e.g. [16], p. 111).



Figure 2. An electron e is scattered by a charged particle Q (see text). 
The energy-momentum exchanged in the scattering example of Figure 2 applies to cases where the incoming particle is not destroyed in the process. Therefore, it is also relevant to analogous cases of weak interactions of neutrino scattering. Hence, like in the electron scattering of Figure 2, also in the case of neutrino scattering, no genuinely real particle is exchanged between the colliding particles.

This subsection shows the usefulness of the constraints of Section 2. It presents several examples that demonstrate that electromagnetic radiation fields are not connected to a specific charge of the radiating system, and that electromagnetic radiation fields and electromagnetic bound fields are different physical objects.

\subsection{Fields of Massive Particles Having Integral Spin}

Let us examine the Lagrangian density of a Klein-Gordon (KG) particle and the more complicated Lagrangian density of the $W^{ \pm}, Z$ and the Higgs particles. The Lagrangian density of each of these particles has a term that is a product of two derivatives of the quantum function, like (see [15], p. 715; [17], p. 198)

$$
\mathcal{L}=\phi_{, \mu}^{\dagger} \phi_{, v} g^{\mu v}+\cdots
$$

or other kinds of a product of two first-order derivatives with respect to the space-time coordinates (see [13], p. 518). The dimension of a Lagrangian density is $\left[L^{-4}\right]$. Therefore, the Lagrangian density (16) proves that the dimension of the product $\phi^{\dagger} \phi$ of each of these particles is $\left[L^{-2}\right]$. It follows that the quantum functions of the KG, $W^{ \pm}, Z$ and the Higgs particles are inherently different from that of a Dirac function, where the dimension of $\psi^{\dagger} \psi$ is $\left[L^{-3}\right]$. Density has the dimension $\left[L^{-3}\right]$. Therefore, unlike the Dirac expression for density (11), an expression for density of these particles must contain a derivative. The constraint 7 shows that an expression of density that depends on derivatives is unacceptable. This outcome means that the KG, $W^{ \pm}, Z$ and the Higgs particles have an inherently erroneous structure. Specific problems that stem from this discrepancy are discussed below in Section 4 .

\subsection{Mathematically Real Quantum Fields of a Massive Particle}

Let us examine massive quantum particles that are described by mathematically real functions: the KG real particle (see e.g. [12], p. 26; [15], pp. 16, 17), the $Z$ boson (see [15], pp. 701, 702), the Higgs boson (see [15], p. 715); the Majorana neutrino (see [20], p. 486); and the Proca massive photon (see [18], pp. 597-601).

An examination of the Noether theorem for the 4-current $j^{\mu}$ (7) shows that it depends on the invariance of the Lagrangian density with respect a global complex phase factor $\exp (i \alpha)$ of the quantum function. Evidently, a transformation that depends on this complex phase factor does not apply to a mathematically real function. Hence, the standard proof of a conserved 4-current does not hold for mathematically real quantum functions. In particular, there is no proof for a consistent expression for density, which is the 0 -component of $j^{\mu}$. 
In the case of the real KG particle, the literature shows an explicit proof of the absence of density and of a conserved 4-current for this quantum field (see [21], p. 42). Analogous problems hold for the Majorana fermion. Here every $\gamma$ matrix of the Dirac theory takes a pure imaginary form (see [20], p. 486). This representation cast the Dirac equation of a free particle into this real form

$$
\left(\gamma^{\mu} i \partial_{\mu}-m\right) \psi
$$

The following serious problems hold for the Majorana form of the Dirac equation. Let us examine the Majorana Lagrangian density

$$
\mathcal{L}=\bar{\psi}\left[\gamma^{\mu} i \partial_{\mu}-m\right] \psi
$$

By definition $\bar{\psi} \equiv \psi^{\dagger} \gamma^{0}$. Hence, the pure imaginary $\gamma^{0}$ means that also the Majorana Lagrangian density (18) and its action (2) are pure imaginary. Furthermore, another problem of this representation is related to the corresponding Hamiltonian, which is written not in terms of $\bar{\psi}$, but in terms of $\psi^{\dagger}$ (see [12], p. 87; [15], p. 52). Hence, the pure imaginary $\gamma^{0}$ of Majorana means that the Majorana Hamiltonian is anti-Hermitian and its eigenvalues are pure imaginary. This is unacceptable because the Hamiltonian represents the system's energy, which is described by a real number.

Analogous problems exist with the $Z$ and the Higgs bosons. The fact that textbooks do not show a consistent expression for density of these particles indicates that this problem is unsettled. Referring to the Proca idea of a massive photon, experiment is consistent with Maxwellian electrodynamics where the photon is a massless particle. The present upper bound of the photon mass is 24 orders of magnitude smaller than the electronic mass [6]. This evidence means that the existence of a Proca photon is extremely unlikely.

The following general proof substantiates the foregoing arguments. The de Broglie principle 3 means that a free quantum particle has a phase whose form is described by one of the following factors of its wave functions (see [9], p. 18)

$$
\cos (k x-\omega t), \sin (k x-\omega t), \mathrm{e}^{ \pm(k x-\omega t)} .
$$

Only the first and the second functions of (19), contribute to a mathematically real quantum function. Hence, simple trigonometry proves that the general form of the required mathematically real phase factor is

$$
\Phi(t, x)=A \sin (k x-\omega t-\delta),
$$

where $A, \delta$ are mathematically real quantities which are a normalization factor and a phase displacement term, respectively.

Let us examine a massive particle in its rest frame. Here $k=0$ and (20) reduces to this form

$$
\Phi(t, x)=-A \sin (\omega t+\delta) .
$$

This result means that for every integer $n$, there is an instant $t$ when $\omega t+\delta=n \pi$. At this instant the quantum function (21) vanishes throughout the entire three-dimensional space, and the particle disappears. It follows that den- 
sity cannot be defined for a massive quantum particle whose wave function takes a mathematically real form.

\section{Discussion}

The previous sections contain an examination of some general laws of quantum fields. The following lines discuss problematic properties of specific issues that are relevant to the laws mentioned above.

D1) The present form of the QED Lagrangian density comprises three terms

$$
\mathcal{L}_{\text {EMW }}=\bar{\psi}\left(\gamma^{\mu} i \partial_{\mu}-m\right) \psi-\frac{1}{16 \pi} F^{\mu v} F_{\mu \nu}-e \bar{\psi} \gamma^{\mu} A_{\mu} \psi
$$

Here the first term represents a free Dirac particle, the second term represents free electromagnetic fields and the third term represents electromagnetic interaction of a charged Dirac particle (see [12], p. 84; [15], p. 78).

The electromagnetic field $F^{\mu v}$ of (22) is a sum of bound fields and radiation fields. Hence, the quadratic form of the second term of this Lagrangian density contains a product of bound fields and radiation fields

$$
-\frac{1}{16 \pi} F^{\mu v} F_{\mu \nu}=-\frac{1}{16 \pi} F_{R}^{\mu \nu} F_{R \mu \nu}-\frac{1}{16 \pi} F_{B}^{\mu v} F_{B \mu \nu}-\frac{1}{8 \pi} F_{R}^{\mu \nu} F_{B \mu \nu},
$$

where the subscripts ${ }_{R, B}$ denote radiation fields and bound fields, respectively. The analysis of subsection 3.2 proves that the last term of (23) should be removed, because it combines two inherently different quantities.

D.2) Let us examine fields that represent electrically charged particles that have an integral spin, like the charged KG particles and the electroweak $W^{ \pm}$ particles. As explained in 3.3, the 4-current of these fields contains a derivative. For this reason, the standard prescription (12) for electromagnetic interactions leads to inconsistent results where the Lagrangian density contains terms that are quadratic in the electromagnetic 4-potential $A_{\mu}$ (For the KG particle, see [15] pp. 690, 691; [17], p. 198; for the $W^{ \pm}$see [13], p. 518).

These expressions are alien to the Lagrangian density of Maxwellian electrodynamics, which depends linearly on the electromagnetic 4-potential $A_{\mu}$ (22). This contradiction means that each of the present theories of elementary massive charged boson-the charged KG particle and the electroweak $W^{ \pm}$particles-violates Maxwellian electrodynamics.

The actual status of the electromagnetic interaction of the $W^{ \pm}$provides an indication of the correctness of the above mentioned contradiction. Here one finds that instead of a theoretically solid expression for this interaction, people use an effective expression for this purpose. Thus, the quantity $W_{\mu \nu}=\partial_{\mu} W_{v}-\partial_{\nu} W_{\mu}$ is used in Equation (2.1) of [22]. Hence, the Noether expression for density (8) proves that this interaction modifies the particle's density. This detrimental effect means that the scalar product of the Hilbert space and of the associated Fock space are destroyed, and quantum operators become meaningless. The same problem exists with calculations published by teams of modern accelerators that use this formula [23] [24]. 
It is interesting to mention the following evidence. The electroweak theory of the charged $W^{ \pm}$bosons is about fifty years old and it still uses effective expressions for its charge density. By contrast, a consistent expression for a conserved 4 -current of the Dirac equation of the electron (and of its associated density) was found about one month after the publication of this equation [25] [26]. This comparison indicates that the fact that consistent expressions for a conserved 4-current of the $W^{ \pm}, Z$ electroweak particles and of the Higgs boson have not been found after about 50 years means that the existence of these expressions is extremely unlikely.

D.3) Requirement 8 emphasizes the crucial role of the equations of motion that are derived from the fields' Lagrangian density. It turns out that unlike Maxwellian electrodynamics and the Dirac theory of massive spin- $1 / 2$ particles, textbooks do not show an explicit form of the equation of motion of the $W^{ \pm}, Z$ and the Higgs bosons. A fortiori, no consistent solution of these equations is presented. The omission of this crucial element is an indication of the above mentioned contradictions of quantum theories of these particles.

\section{Conclusions}

This work is based on a fundamental principle of physics which states that an acceptable physical theory must take a consistent mathematical structure. The second section explains why a quantum field theory should abide by the following requirements:

- It takes a relativistically covariant form.

- It should be derived from the variational principle.

- A free quantum particle should satisfy the de Broglie principle.

- Properties of a quantum particle should be consistent with the results of Wigner's analysis of the Poincare group.

- Its Lagrangian density must contain a consistent interaction term.

- Correspondence relations should hold between theories.

- A massive particle must have a consistent expression for density.

- The Lagrangian density of a quantum particle must yield partial differential equations whose solutions adequately describe the particle's experimental data.

These requirements are used for a derivation of some general laws. For example, a straightforward application of the de Broglie law proves that a mathematically real function cannot describe an elementary massive quantum particle. Hence, the present descriptions of the $Z$ boson, the Higgs particle, the neutral KG boson, the Majorana neutrino and the Proca massive photon are unjustified. A further examination shows other inconsistencies of the quantum theories of the $W^{ \pm}, Z$ bosons, the Higgs boson and the KG particles. Furthermore, some changes should be introduced into the Lagrangian density of electromagnetic fields.

\section{Conflicts of Interest}

The author declares no conflicts of interest regarding the publication of this paper. 


\section{References}

[1] Wigner, E. (1939) On Unitary Representations of the Inhomogeneous Lorentz Group. Annals of Mathematics, 40, 149-204. https://doi.org/10.2307/1968551

[2] Weinberg, S. (1995) The Quantum Theory of Fields, Vol. I. Cambridge University Press, Cambridge. https://doi.org/10.1017/CBO9781139644167

[3] Schweber, S.S. (1964) An Introduction to Relativistic Quantum Field Theory. Harper \& Row, New York, 44-53.

[4] Sternberg, S. (1994) Group Theory and Physics. Cambridge University Press, Cambridge, 143-150.

[5] Dehmelt, H. (1988) A Single Atomic Particle Forever Floating at Rest in Free Space: New Value for Electron Radius. Physica Scripta, T22, 102. https://doi.org/10.1088/0031-8949/1988/T22/016

[6] Tanabashi, M., et al. (2018) Review of Particle Physics. Physical Review D, 98, Article ID: 030001.

[7] Griffiths, D. (2008) Introduction to Elementary Particles. 2nd Edition, Wiley-VCH, Weinheim.

[8] Messiah, A. (1967) Quantum Mechanics. Vol. 1, North Holland, Amsterdam.

[9] Schiff, L.I. (1955) Quantum Mechanics. McGraw-Hill, New York.

[10] Dirac, P.A.M. (1958) The Principles of Quantum Mechanics. Oxford University Press, London. https://doi.org/10.1063/1.3062610

[11] Halzen, F. and Martin, A.D. (1984) Quarks and Leptons: An Introductory Course in Modern Particle Physics. John Wiley, New York.

[12] Bjorken, J.D. and Drell, S.D. (1965) Relativistic Quantum Fields. McGraw-Hill, New York. https://doi.org/10.1063/1.3047288

[13] Sterman, G. (1993) An Introduction to Quantum Field Theory. Cambridge University Press, Cambridge. https://doi.org/10.1017/CBO9780511622618

[14] Landau, L.D. and Lifshitz, E.M. (2005) The Classical Theory of Fields. Elsevier, Amsterdam.

[15] Peskin, M.E. and Schroeder, D.V. (1995) An Introduction to Quantum Field Theory. Addison-Wesley, Reading.

[16] Bjorken, J.D. and Drell, S.D. (1964) Relativistic Quantum Mechanics. McGraw-Hill, New York.

[17] Pauli, W. and Weisskopf, V. (1934) Uber die Quantisierung der skalaren relativistischen Wellengleichung. Helvetica Physica Acta, 7, 709-731.

Miller, A.I. (1994) Early Quantum Electrodynamics. University Press, Cambridge, 188-205.

[18] Jackson, J.D. (1975) Classical Electrodynamics. 2nd Edition, John Wiley, New York.

[19] Perkins, D.H. (1987) Introduction to High Energy Physics. Addison-Wesley, Menlo Park.

[20] Pal, P.B. (2011) Dirac, Majorana, and Weyl Fermions. American Journal of Physics, 79, 485. https://doi.org/10.1119/1.3549729

[21] Berestetskii, V.B., Lifshitz, E.M. and Pitaevskii, L.P. (1982) Quantum Electrodynamics. Pergamon, Oxford. https://doi.org/10.1016/B978-0-08-050346-2.50020-9

[22] Hagiwara, K., Peccei, R.D., Zeppenfeld, D. and Hikaso, K. (1987) Probing the Weak Boson Sector in $\mathrm{e}^{+} \mathrm{e}^{-} \rightarrow \mathrm{W}^{+} \mathrm{W}^{-}$. Nuclear Physics B, 282, 253-307.

https://doi.org/10.1016/0550-3213(87)90685-7 
[23] Abazov, V.M., et al. (2012) Limits on Anomalous Trilinear Gauge Boson Couplings from WW, WZ and W $\gamma$ Production in Collisions at $\sqrt{\mathrm{s}}_{\mathrm{s}}=1.96 \mathrm{TeV}$. Physics Letters $B$, 718, 451-459.

[24] Aad, G., et al. (2012) Measurement of the WW Cross Section in pp Collisions with the ATLAS Detector and Limits on Anomalous Gauge Couplings. Physics Letters B, 712, 289-308.

[25] Dirac, P.A.M. (1928) Quantum Theory of the Electron. Proceedings of the Royal Society of London. Series A, 117, 610-624.

[26] Darwin, C.G. (1928) The Wave Equations of the Electron. Proceedings of the Royal Society of London. Series A, 118, 654. 